ORIGINAL PAPER

\title{
SEROUS VERSUS HIGH-GRADE ENDOMETRIOID ENDOMETRIAL CARCINOMA: IMMUNOHISTOCHEMISTRY OF RFP IS NOT USEFUL FOR DIFFERENTIATION
}

\author{
Cigdem Ussakli ${ }^{1}$, Alp Usubutun ${ }^{1}$, Nazmiye Dincer ${ }^{2}$, Anil Dolgun ${ }^{3}$, Dillek Bülbül ${ }^{3}$, \\ Zuhal Isikdogan ${ }^{4}$, Nihan Haberal ${ }^{5}$, Ozlem Ozen ${ }^{5}$, Gaye Guler Tezel ${ }^{1}$
}

\author{
${ }^{1}$ Department of Pathology, Hacettepe University, Ankara, Turkey \\ ${ }^{2}$ Department of Pathology, Ankara Atatürk Training and Research Hospital, Ankara, Turkey \\ ${ }^{3}$ Department of Biostatistics, Hacettepe University, Ankara, Turkey \\ ${ }^{4}$ Department of Pathology, Etlik Zübeyde Hanim Research and Teaching Hospital, Ankara, Turkey \\ ${ }^{5}$ Department of Pathology, Başkent University, Ankara, Turkey
}

\begin{abstract}
We evaluated the immunohistochemical expression of ret finger protein (RFP) along with conventional immunohistochemical markers in endometrioid and serous carcinomas of the endometrium.

A total of 124 endometrial carcinoma cases (24 grade 1 endometrioid, 60 grade 3 endometrioid, 40 serous) were retrieved from pathology archives. Tissue microarrays were constructed. The expression of RFP, WT1, ER, PR, p53 and p16 was examined immunohistochemically. Sensitivity, specificity, area under the receiver operating characteristic (ROC) curve, $\kappa$ statistic for interobserver reproducibility, Kruskal-Wallis test, Mann-Whitney U test and Fisher's exact tests were performed for statistical analyses. The mean RFP score was 1.54 in grade 1 endometrioid, 4.31 in grade 3 endometrioid, and 6.31 in serous carcinomas ( $\mathrm{p}<0.001$ ). Overall, RFP scores were higher both in serous and grade 3 endometrioid carcinoma ( $p>0.05$ ), and significantly lower in grade 1 endometrioid carcinoma ( $<<0.05)$. p16 and p53 staining patterns were able to differentiate between high-grade endometrioid and serous carcinoma $(p<0.001)$. ER, $\mathrm{PR}$ and WT-1 did not reach statistical significance for subtyping. The $\kappa$ values of the general agreement between the observers were 0.737 and 0.727 for endometrioid and serous carcinomas respectively ( $\mathrm{p}<0.001)$.

Diffuse $\mathrm{p} 53$ and $\mathrm{p} 16$ staining provides the most sensitive and specific immunomarkers for differentiating high-grade endometrioid and serous carcinomas.
\end{abstract}

Key words: endometrial neoplasms, immunohistochemistry, RFP, serous carcinoma, endometrioid carcinoma.

\section{Introduction}

Endometrial cancer is the fourth most common malignancy among women in the US and the fifth leading cause of cancer-related deaths in women in western countries [1]. Endometrial carcinoma is a morphologically heterogeneous disease with several histotypes and grades. These histotypes, when taken together with their clinical features, can be divided into two major categories. Type 1 endometrial carcinomas are histologically low-grade endometrioid type adenocarcinomas (EAC). They have a good prognosis, are associated with unopposed estrogen exposure, and are related to endometrial hyperplasia $[2,3]$. Type 2 endometrial carcinomas (uterine serous carcinomas [USC], clear 
cell carcinomas [CCC] and carcinosarcomas) are histologically high-grade lesions, of non-endometrioid morphology, unrelated to estrogen excess, and have a less favorable prognosis than type 1 carcinomas [4]. Type 1 carcinomas are usually diagnosed in the earlier stages, and their management is less drastic [5]. Type 2 cancers, although less frequently seen, account for a disproportionately high number of deaths when compared to type 1 cancers $[6,7]$.

The molecular background of these two groups is dissimilar $[2,8]$. Integrated genomic and proteomic analysis of endometrial carcinomas can reveal totally different subtype groupings and may shed light on future classifications [9]. In daily practice, to address this problem of differentiating the two types of endometrial malignancy, a large number of immunomarkers have been proposed and evaluated [10, 11, 12].

Ret finger protein (RFP) was originally identified as a fusion protein with RET receptor tyrosine kinase and exhibits transcriptional repressive activity [13]. This protein belongs to the large B-box RING finger protein family, which has more than 200 members. RFP is expressed in several tumor cell lines of rodents and humans $[13]$, but the exact function of this protein in tumors is not clear. A previous study has shown that RFP expression might be helpful in differentiating serous carcinomas and (type 1) endometrial carcinomas [14]. Immunohistochemical expression of RFP may be associated with adverse outcomes - in terms of overall survival and progression-free survival - in endometrial cancer patients, irrespective of histological type [15].

This study aims to determine and compare the immunohistochemical expression of RFP with conventional immunohistochemical markers (p16, WT1, p53, PR, ER) in grade 1 and grade 3 EAC and USC of the endometrium. The hypothesis is that RFP may aid in the distinction of high-grade endometrial carcinomas of endometrioid type from serous carcinomas of the endometrium.

\section{Material and methods}

\section{Case selection and tissue microarray construction}

This study was performed retrospectively on samples of hysterectomy specimens, dating from January 2000 to December 2010, retrieved from the pathology archives of three institutions (Department of Pathology, Hacettepe University; Department of Pathology, Ministry of Health Etlik Zubeyde Hanim Women's Health Education and Research Hospital; Department of Pathology, Baskent University).

Cases with a diagnosis of high-grade endometrial EAC (grade 3) and USC were selected, as well as 24 cases of grade 1 endometrial EAC from the archives of Hacettepe University Hospital. In the previous study [14], serous carcinomas were positive and low-grade endometrioid carcinomas were negative with RFP, so we selected grade 1 EAC cases to compare the RFP expression with grade 3 EAC. All cases were reevaluated and classified according to the World Health Organization (WHO) system, and a tumor grade was given based on the FIGO grading system. A total of 124 endometrial carcinoma cases (60 grade 3 EAC, 40 USC, 24 grade 1 EAC) were selected by five experienced gynecopathologists from the 3 institutions. Representative slides and paraffin blocks were chosen and tissue microarrays were constructed. The pathologists also independently reviewed immunohistochemically stained slides, assessing scores for the expression of each immunohistochemical marker and noting the final diagnosis for each case. The patients' ages, the presence or absence of ovarian involvement by carcinoma, and FIGO stages were also noted.

Paraffin-embedded tissues from 124 endometrial adenocarcinomas and control tissues were used to construct a tissue microarray. Biopsies from non-neoplastic endometrial tissues from separate cases of patients were used as normal controls. Briefly, after the morphologically representative region on the blocks was chosen (donor block), a core tissue biopsy of 0.6 $\mathrm{mm}$ was punched and transferred to the recipient block (receiver block). To overcome tumor heterogeneity and loss of tissue, three cores were selected from each case, representing different areas of each tumor. One section per block was stained with hematoxylin and eosin (HE).

\section{Immunohistochemistry}

Immunohistochemical studies were carried out manually. Sections of representative tumor (USC and grade 3 EAC) blocks, 4 microns thick, were stained with HE, RFP, WT1, ER, PR, p53 and p16. Lowgrade endometrioid tumors usually can easily be differentiated from high-grade tumors by $\mathrm{HE}$ stained slides. Because of this fact, low-grade EAC cases were only stained with FFP to search for the role in endometrial carcinogenesis. Immunohistochemical staining was carried out by the strepto-avidin biotin method, using commercially available kits for WT1 (1/50 Biocare, USA), ER (1/100 Thermo, USA), PR (1/300, Neomarker, USA), p53 (1/800 Leica, UK), and p16 (1/75 Biocare, USA). Anti-RFP antibody (IBL Co, Japan) was used as a primary antibody [16]. Briefly, deparaffinized sections were treated with methanol containing $0.3 \% \mathrm{H}_{2} \mathrm{O}_{2}$ for $15 \mathrm{~min}$ for endogenous peroxide blockage. After washing with PBS, blocking solution was applied for 5 minutes. All slides received pretreatment with citrate for antigen retrieval. Then, anti-RFP primary antibody was allowed to react at room temperature for 60 minutes in a dilution of $1: 750$. After washing in PBS, biotinylated antirabbit IgG goat secondary antibody was applied for 10 minutes, followed by horseradish peroxidase-marked 
strepto-avidin for 10 minutes. Peroxidase was visualized by diaminobenzidine tetrahydrochloride containing $0.3 \% \mathrm{H}_{2} \mathrm{O}_{2}$. Nuclei were stained with Harris' hematoxylin. Appropriate controls were included for this study. The RFP expression was evaluated as positive when there was nuclear staining.

For statistical analysis, a semi-quantitative scoring method (percentage $\times$ intensity) was used, and ranged from 0 to 15 , as previously described [14]. The percentage of staining (scored on a scale of 0-5) and staining intensity (scored on a scale of + to +++ ; with $0=$ negative, $+=$ mild, $++=\bmod -$ erate, $+++=$ severe) were assessed. The percentage of staining was scored as follows: $0=0 \% ; 1=$ $1-5 \% ; 2=6-25 \% ; 3=25-50 \% ; 4=50-75 \%$; and $5=75-100 \%$. The scoring of immunohistochemistry was done using a compositional method (intensity $\times$ percentage of staining) for all the markers. P16 and p53 staining was also evaluated in a two-tier system where diffuse staining in almost $100 \%$ of tumor cells was separately noted. Additionally, p16 and p53 staining was evaluated in a two-tier system where severe staining of cells in more than $95 \%$ of the cell population categorized group 1 and the others having less staining than $95 \%$ of the cell population constituted group 2 .

\section{Statistical analyses}

For each marker tested, sensitivity and specificity were calculated. Sensitivity is the probability of correctly identifying a true positive result using the test, and specificity is the probability of correctly identifying a true negative result. An ideal test would have a sensitivity and specificity score of 1 , i.e. no false positives and no false negatives. Traditionally, a tradeoff is often needed between the two measures and, depending on the case, higher sensitivity or higher specificity may be more desirable. In our case, we argued that a higher specificity reduces the chance of misclassifying the tumor as EAC, and is therefore more useful. Only a p-value of $<0.05$ was considered to be significant. After determining the expression of each immunomarker in endometrial cancer, we analyzed the value of each marker, or any combination of them, in differentiating EAC from USC. Sensitivity, specificity, area under the receiver operating characteristic (ROC) curve, $\kappa$ statistic for interobserver reproducibility, Kruskal-Wallis test, Mann-Whitney U test and Fisher's exact tests were performed for the statistical analyses. IBM SPSS Statistics for Windows, Version 20.0 (Armonk, NY: IBM Corp, Released 2011) was used for all analyses.

\section{Results}

The patients' ages ranged from 28 to 89 years (mean, 64.79 for grade 1 EAC; 60.91 for grade 3 EAC; 65.93 for USC; 64.09 for all cases). The study consisted of 124 endometrial carcinomas with 60 grade 3 EAC, 40 USC, and 24 grade 1 EAC. The high-grade cases were distributed as 31 stage I (18 USC, 13 EAC), 12 stage II (6 USC, 6 EAC), 29 stage III (19 USC, 10 EAC), and 13 stage IV (11 USC, 2 EAC) tumors. We were unable to obtain detailed clinical information for 15 patients.

Interobserver reproducibility was evaluated and the study was designed according to the consensus diagnosis. A total of 100 high-grade endometrial tumor cases (60 cases of grade 3 EAC and 40 cases of USC) were reevaluated. As mentioned earlier, all slides were evaluated independently by five pathologists and the general agreement between the observers was evaluated. The pathologists categorized the tumors as EAC and USC. The $\kappa$ values were 0.737 and 0.727 for EAC and USC, respectively ( $\mathrm{p}<0.001)$. Overall agreement was 0.713 ( $\mathrm{p}<0.001)$.

The distribution of p16, p53, WT1, ER, PR and RFP expression scores according to the diagnosis is summarized in Table I. The RFP scores in each histologic type were compared: the mean RFP score was 1.54 in grade 1 EAC, 4.31 in grade 3 EAC and 6.31

Table I. Immunohistochemical markers' scores (p16, PR, ER, RFP, p53, WT-1) according to tumor types

\begin{tabular}{lccccccc}
\hline TUMOR TYPE & & RFP SCORE & P16 SCORE & PR SCORE & WT-1 SCORE & P53 SCORE & ER SCORE \\
\hline EAC & Number of cases & 24 & NA & NA & NA & NA & NA \\
grade 1 & mean (median) & $1.54(1)$ & NA & NA & NA & NA & \\
\hline EAC & Number of cases & 35 & 33 & 36 & 34 & 36 & 37 \\
grade 3 & mean (median) & $4.31(4)$ & $10.82(12)$ & $5.67(1)$ & $2.21(1)$ & $6.22(5)$ & $5.81(4)$ \\
\hline USC & Number of cases & 57 & 57 & 56 & 55 & 57 & 57 \\
& mean (median) & $6.31(6)$ & $13.32(15)$ & $3.45(1)$ & $3.11(1)$ & $11.49(15)$ & $4.81(1)$ \\
\hline TOTAL & Number of cases & 92 & 90 & 92 & 89 & 93 & 94 \\
& mean (median) & $5.45(4)$ & $12.40(15)$ & $4.32(1)$ & $2.76(1)$ & $9.45(12)$ & $5.20(1)$ \\
\hline
\end{tabular}

*The number of cases differs because some cases cannot be evaluated as the result of technical problems such as sloughing of tissue 
Table II. Sensitivity and specificity of immunohistochemical markers differentiating high-grade endometrial tumors

\begin{tabular}{lccccc}
\hline SCORE & Best CUT-OFF & Sensitivity & SPeCIficity & ROC & P VALUE \\
\hline p16 & 13.5 & 0.8 & 0.571 & 0.684 & 0.003 \\
\hline p16 diffuse & 1.5 & 0.69 & 0.83 & 0.765 & $<0.001$ \\
\hline p53 & 13.5 & 0.69 & 0.84 & 0.745 & $<0.001$ \\
\hline p53 diffuse & 13.5 & 0.84 & 0.62 & 0.735 & $<0.001$ \\
\hline WT1 & & & 0.588 & 0.163 \\
\hline ER & & & 0.558 & 0.339 \\
\hline PR & & & 0.598 & 0.113 \\
\hline RFP & & & 0.618 & 0.058 \\
\hline
\end{tabular}

Note that in Table II we did not report a best cut-off for sensitivity and specificity in WT1, ER, PR and RFP markers because the area under the ROC curve is not statistically significant for these markers.

Table III. Area under the ROC curve

\begin{tabular}{ccc}
\hline IMMUNOMARKER & $\begin{array}{c}\text { AREA UNDER } \\
\text { THE ROC CURVE }\end{array}$ & P VALUE \\
\hline p16 & 0.682 & 0.006 \\
\hline WT-1 & 0.587 & 0.192 \\
\hline p53 & 0.774 & $<0.001$ \\
\hline RFP & 0.589 & 0.185 \\
\hline PR & 0.559 & 0.345 \\
\hline ER & 0.543 & 0.496 \\
\hline
\end{tabular}

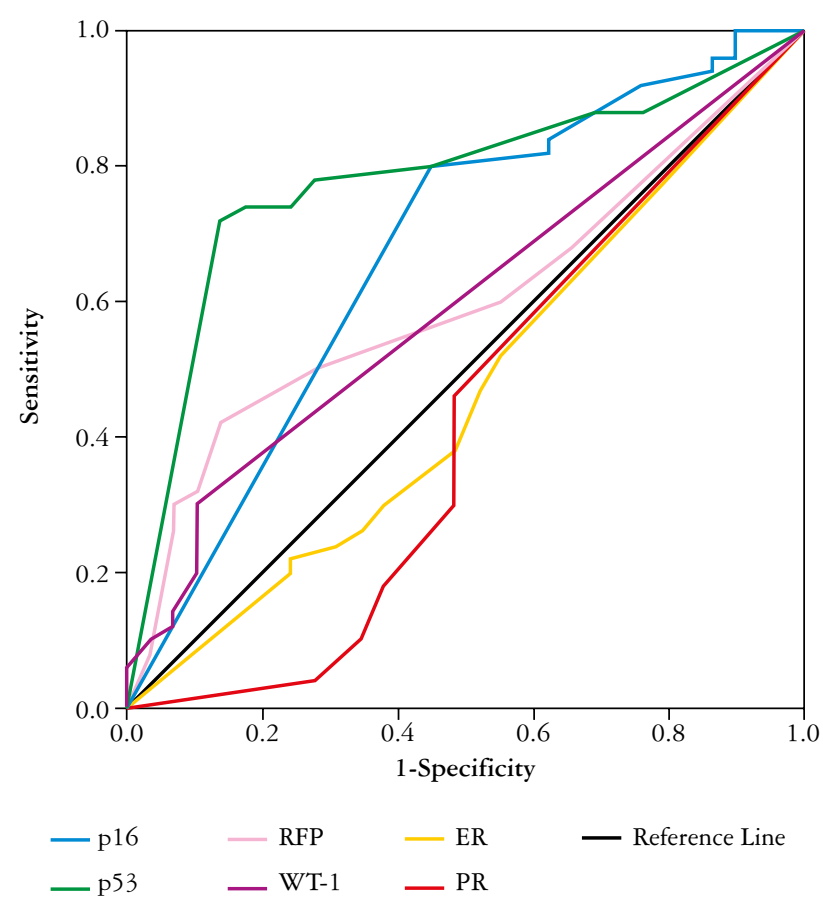

Fig. 1. ROC curves for predicting high-grade EAC and USC using p53, p16, RFP, WT-1, PR and ER. According to the ROC curves, $\mathrm{p} 53$ and p16 are statistically significant markers for discriminating high-grade EAC $(\mathrm{p}<0.001$ and $\mathrm{p}=0.006$, respectively) in USC ( $\mathrm{p}<0.001)$. Overall, RFP scores were higher in both USC and grade 3 EAC cases ( $p>0.05)$, whereas they were significantly lower in grade 1 EAC $(\mathrm{p}<0.05)$ (Table I).

We evaluated the sensitivity and specificity of the immunohistochemical markers for differentiating EAC and USC (Table II). We found that p16 and p53 staining patterns can differentiate between highgrade EAC and USC. A discrimination score was obtained for p16 and p53, which was found to be statistically significant $(\mathrm{p}=0.003$ and $\mathrm{p}<0.001$, respectively).

We examined the utility of the markers in determining the tumor types. According to the ROC curves predicting high-grade EAC and USC using immunomarkers, p53 and p16 were the most useful markers for differentiating/discriminating the different histologic types (Table III, Figs. 1 and 2).

Note that in Fig. 1, for p16, p53, RFP and WT-1 markers, higher test results indicate high-grade endometrial tumors, whereas for PR and ER markers lower test results indicate high-grade endometrial tumors. Therefore, when we include all markers in the same figure, the ROC curves for PR and ER remain under the reference line (the line that indicates there is no discrimination).

Statistically, diffuse p 16 and p 53 staining emerged as the most sensitive (74\% and $74 \%$, respectively) and specific ( $88 \%$ and $92 \%$, respectively) markers for the differentiation of USC from grade 3 EAC $(p<$ 0.001). ER, PR, WT-1 and RFP did not reach statistical significance in the subtyping of high-grade tumors, whereas the RFP score was found to be significantly higher in grade $3 \mathrm{EAC}$ than in grade 1 $(\mathrm{p}<0.001)$.

The RFP score was found to be significantly higher in grade 3 EAC and USC. The area under the ROC curve for USC and grade 3 EAC was 0.597 and was not found to be statistically significant. However, the ROC analysis result was 0.776 for serous versus 

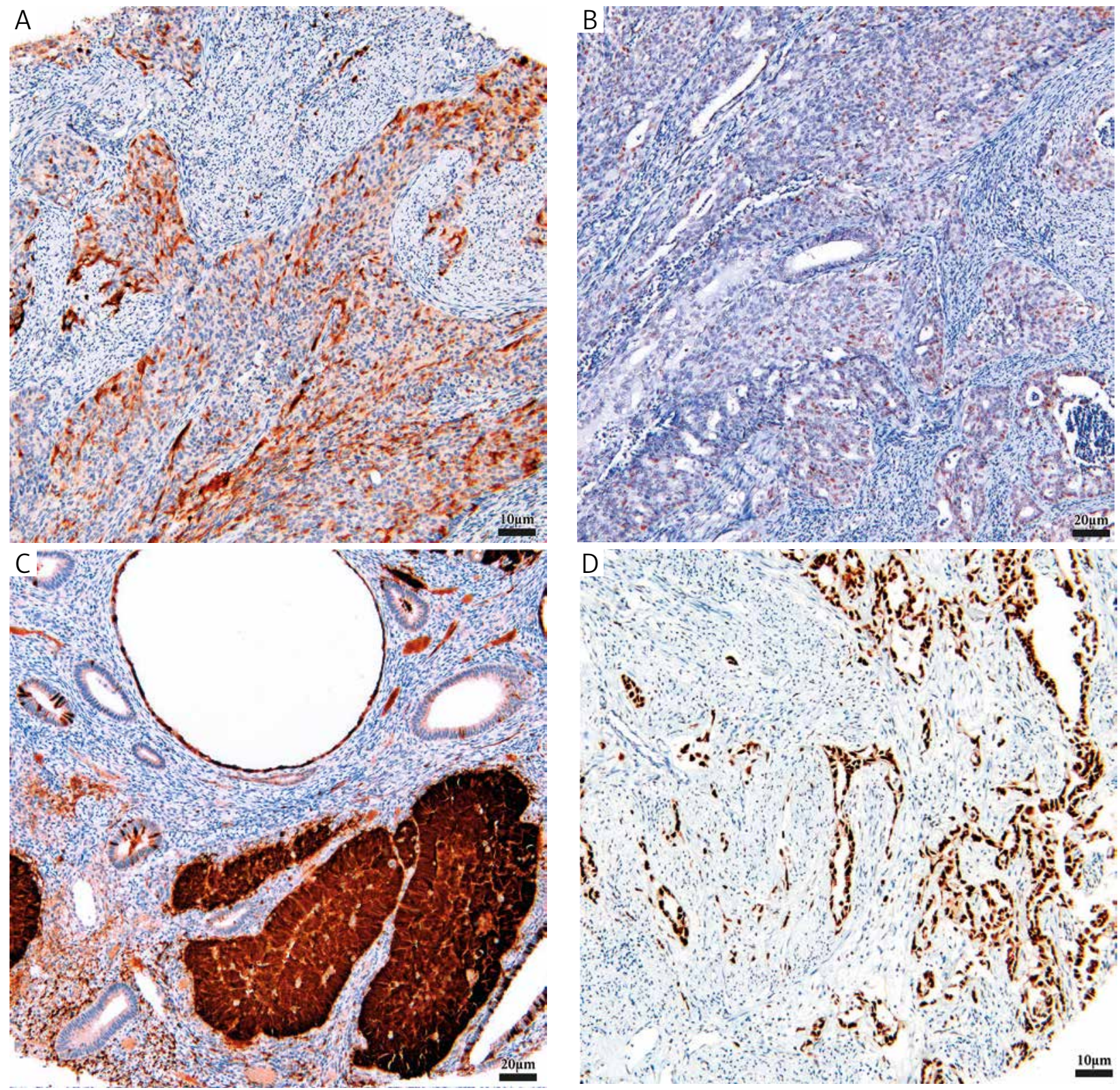

Fig. 2. Immunohistochemical features of p16 and p53 in high-grade EAC and USC. A) p16 in EAC; B) p53 in EAC; C) p16 in USC and D) p53 in USC

grade $1 \mathrm{EAC}$, and 0.44 for grade $3 \mathrm{EAC}$ versus grade 1 EAC ( $p<0.001$ and $p=0.002$, respectively). Different RFP expression patterns can be seen in Fig. 3.

\section{Discussion}

Differentiating EAC from USC and CCC is important for patient treatment and prognosis. It is also imperative to confirm an accurate diagnosis, not only on hysterectomy specimens but also on biopsy/curetting specimens, in order to plan treatment, which in turn might have an impact on patient management intraoperatively. Although molecular features of endometrial cancers may shed light on the classification of high-grade endometrial cancers [9], we still clas- sify endometrial cancers conventionally according to the morphology of the tumor. Common high-grade endometrial carcinomas include grade 3 EAC, USC and CCC. Most of the high-grade tumors can be diagnosed by their morphological features and classified on the basis of histopathology. However, further evaluation is needed for tumors that cause diagnostic confusion.

In our previous study, we observed that RFP was expressed in all of the ovarian serous carcinoma and USC, whereas it was negative in EAC type endometrial tumors, endometrial intraepithelial neoplasia, and benign endometrial polyps [14]. In our previous study, although all the EAC were low grade, we hypothesized that RFP could be used as a marker 

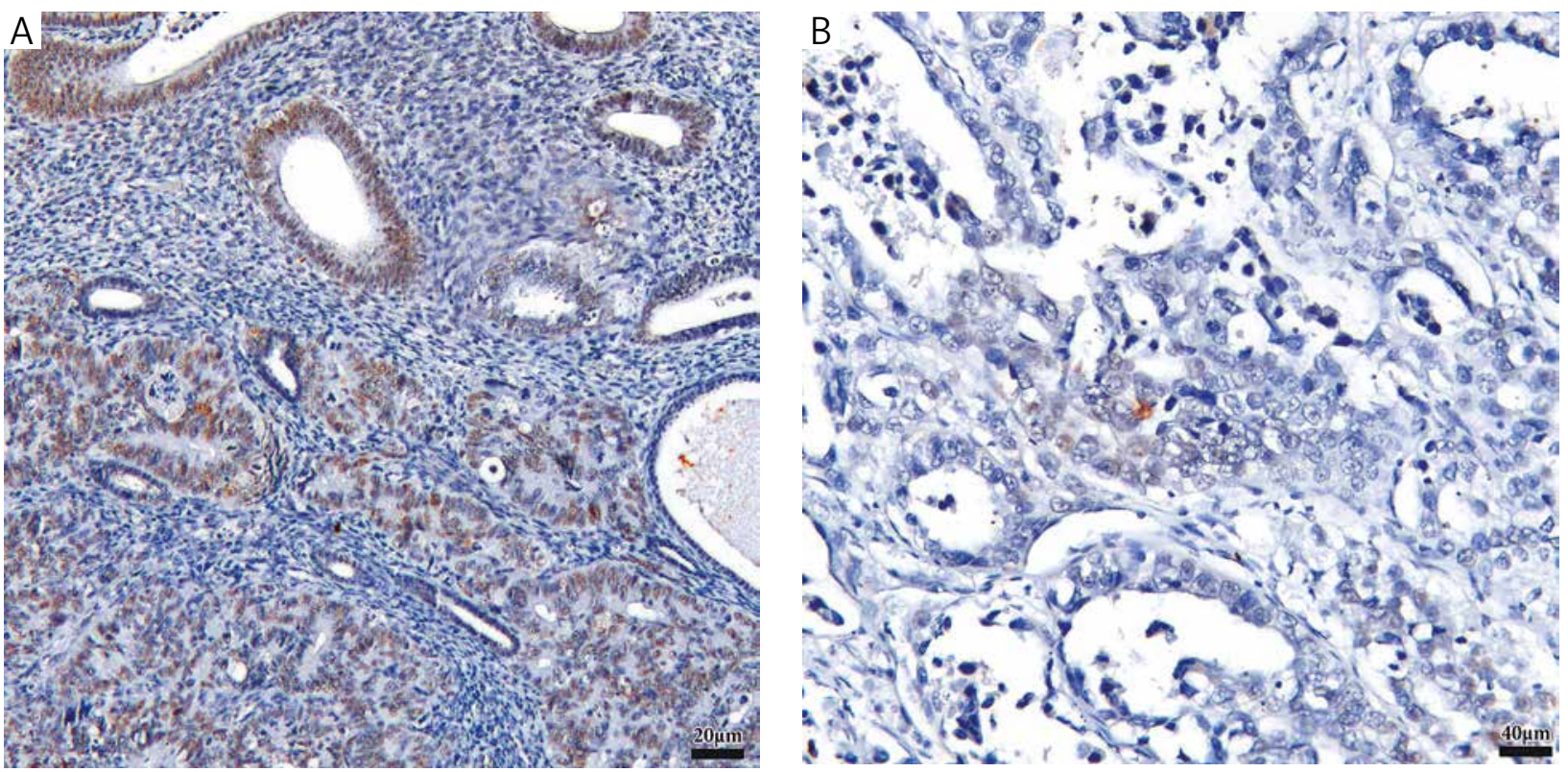

Fig. 3. RFP staining in A) high-grade USC and B) low-grade EAC

to detect USC in difficult cases that exhibit ambiguous features. In addition, RFP was reported to be involved in cancer cell migration in endometrial tumors [15]. In that same study, immunohistochemical expression of RFP was shown to be associated with poor prognosis, such as overall survival and progression-free survival. In the current study, we examined ER, PR, p16, p53, WT-1, and RFP expression in endometrial tumors. These immunohistochemical markers, except RFP, which is not widely used in clinical practice, are well-known adjunct markers in aiding diagnosis in high-grade endometrial tumors $[17$, 18]. We observed that RFP expression is associated both with high-grade EAC and with USC. Because of the similar expression profile of RFP in high-grade carcinomas, this marker cannot be used to differentiate between high-grade EAC and USC. The role of RFP in the carcinogenesis of high-grade endometrial tumors will be a subject of future research projects.

A panel of markers has been used and reported in the literature as being useful in the differential diagnosis of high-grade endometrial tumors, especially when the morphological features are not characteristic. Aberrant p53 expression supports the diagnosis of USC. However, it should be kept in mind that discordant staining patterns can be seen [17]. A small number of grade 2 and 3 EAC overexpress p53, but mainly the expression is focal and patchy. p16 expression is diffuse and strong in USC, whereas staining is absent or scattered in grade 3 EAC [19].

When we compared the usefulness of the immunohistochemical markers listed above for distinguishing USC from high-grade EAC, p16 and p53 emerged as the most valuable markers in our study. Consistent with other studies in the literature, not only the percentage of staining but also the staining intensity is important for this differentiation. In USC, almost all the neoplastic cells stain strongly with both p16 and $\mathrm{p} 53$. Also, deadly negative $\mathrm{p} 53$ staining supports the USC diagnosis. Another interesting finding was that p53 and p16 were the most sensitive and specific markers for differentiating USC from high-grade EAC in this study group. As our study results imply, diffuse (almost all neoplastic cells), strong p53 (or deadly negative) and p16 staining are most consistent with USC rather than with high-grade EAC.

WT-1 is commonly expressed in ovarian serous tumors and accepted as a diagnostic marker for ovarian serous carcinoma. In a previous study, a diverse correlation between WT-1 expression and disease-free survival in USC was noted by the authors [20]. In our current study, there were a few EAC and USC cases staining strongly and diffusely with WT-1, even though most of the endometrial EAC and USC stained poorly or negatively with WT-1.

In our study, there was substantial agreement in the diagnosis of USC and high-grade EAC. However, in one published series evaluating the interobserver and intraobserver agreement, there was a poor concordance rate in differentiating high-grade endometrial cancer [10]. In that study, three experienced gynecologic pathologists evaluated the slides from 59 high-grade endometrial cancer specimens, and a consensus diagnosis was made in only 31 of 59 cases. In another study, the authors examined the reproducibility of the high-grade endometrial cancer diagnosis and used a combination of immunohistochemical markers for a consensus diagnosis. In that study, moderate (k: 0.575$)$ interobserver reproducibility was observed, and the authors suggested using p 53 , 
ER and p16 to distinguish USC from grade 3 EAC. Non-informatively, a triple positive immunoprofile was noted both in grade 3 EAC and USC in similar frequencies. In these situations, the authors again suggested use of additional markers [11]. Hoang et al. reported average $\mathrm{k}$ values (k: 0.55; range: 0.30$0.67)$ for high-grade endometrial cancer in 36 cases. After immunohistochemical evaluation it increased to 0.68 (range: $0.65-0.81$ ) [21].

In our study, we obtained high interobserver agreement among the participating gynecopathologists. It is probably due to the selection of limited cases (only USC and high-grade EAC cases were included, while CCC and undifferentiated carcinomas were excluded) in the study. Distinguishing between EAC and USC is not difficult when the classical histopathological features of each tumor are detected. As our study results supported, in cases where diagnostic disagreements arise, immunohistochemical markers will be helpful. Along with conventional markers, new promising markers such as PTEN and MLH1 proteins, which were intensely positive in USC but not in EAC, may also be helpful [22].

In summary, there are still unresolved problems in the differential diagnosis of highgrade endometrial tumors, one of which is the low reproducibility of morphologic diagnosis. This problem is more common in endometrial carcinomas than in ovarian carcinomas. We do not yet fully understand tumor progression in endometrial cancer, and have limited data for carcinogenesis [17]. In this cohort of highgrade EAC and USC, we were able to reach substantial interobserver agreement and showed that diffuse p53 and p16 staining offers the most sensitive and specific immunomarkers for differentiating these two high-grade tumors. In addition, we confirmed the selective expression of RFP in high-grade endometrial carcinoma compared to grade $1 \mathrm{EAC}$.

\section{The authors declare no conflict of interest.}

\section{References}

1. Siegel R, Naishadham D, Jemal A. Cancer statistics, 2013. CA Cancer J Clin 2013; 63: 11-30.

2. Silverberg SG, Mutter GL, Kurman RJ, et al. Pathology and Genetics of Tumours of the Breast and Female Genital Organs. In: World Health Organization Classification of Tumours. Tavassoli FA, Devilee P. (eds). IARC Press, Lyon 2003; 217-232.

3. Bokhman JV. Two pathogenetic types of endometrial carcinoma. Gynecol Oncol 1983; 15: 10-17.

4. Lax SF. Molecular genetic pathways in various types of endometrial carcinoma: from a phenotypical to a molecular-based classification. Virchows Arch 2004; 444: 213-223.

5. Wright JD, Barrena Medel NI, Sehouli J, et al. Contemporary management of endometrial cancer. Lancet 2012; 379: 1352 1360 .
6. Creasman WT, Kohler MF, Odicino F, et al. Prognosis of papillary serous, clear cell, and grade 3 stage I carcinoma of the endometrium. Gynecol Oncol 2004; 95: 593-596.

7. Halperin R, Zehavi S, Langer R, et al. Uterine papillary serous carcinoma (pure and mixed type) compared with moderately and poorly differentiated endometrioid carcinoma. A clinicopathologic study. Eur J Gynaecol Oncol 2002; 23: 300-304.

8. McConechy MK, Ding J, Cheang MC, et al. Use of mutation profiles to refine the classification of endometrial carcinomas. J Pathol 2012; 228: 20-30.

9. Cancer Genome Atlas Research Network, Kandoth C, Schultz N, et al. Integrated genomic characterization of endometrial carcinoma. Nature 2013; 497: 67-73.

10. Gilks CB, Oliva E, Soslow RA. Poor interobserver reproducibility in the diagnosis of high-grade endometrial carcinoma. Am J Surg Pathol 2013; 37: 874-881.

11. Han G, Sidhu D, Duggan MA, et al. Reproducibility of histological cell type in high-grade endometrial carcinoma. Mod Pathol 2013; 26: 1594-1604.

12. Clarke BA, Gilks CB. Endometrial carcinoma: controversies in histopathological assessment of grade and tumour cell type. J Clin Pathol 2010; 63: 410-415.

13. Takahashi M, Inaguma Y, Hiai H, et al. Developmentally regulated expression of a human "finger"-containing gene encoded by the 5' half of the ret transforming gene. Mol Cell Biol 1988; 8: 1853-1856.

14. Tezel GG, Ordulu Z, Himmetoglu C, et al. The selective expression of ret finger protein in endometrial cancer: can RFP be a marker of serous carcinomas? Turk Patoloji Derg 2012; 28: 213-219.

15. Tsukamoto H, Kato T, Enomoto A, et al. Expression of Ret finger protein correlates with outcomes in endometrial cancer. Cancer Sci 2009; 100: 1895-1901.

16. Tezel G, Nagasaka T, Iwahashi N, et al. Different nuclear/cytoplasmic distributions of RET finger protein in different cell types. Pathol Int 1999; 49: 881-886.

17. Soslow RA. High-grade endometrial carcinomas - strategies for typing. Histopathology 2013; 62: 89-110.

18. Matalka I, Obeidat B, Mohtaseb A, et al. The significance of Wilms Tumor Gene (WT1) and p53 expression in curettage specimens of patients with endometrial carcinomas. Pathol Res Pract 2013; 209: 19-23.

19. Chiang S, Soslow RA. Updates in diagnostic immunohistochemistry in endometrial carcinoma. Semin Diagn Pathol 2014; 31: 205-215.

20. Hedley C, Sriraksa R, Showeil R, et al. The frequency and significance of WT-1 expression in serous endometrial carcinoma. Hum Pathol 2014; 45: 1879-1884.

21. Hoang LN, McConechy MK, Köbel M, et al. Histotype-genotype correlation in 36 high-grade endometrial carcinomas. Am J Surg Pathol 2013; 37: 1421-1432.

22. Bąkiewicz A, Michalak J, Sporny S. Immunoexpression and clinical significance of the PTEN and MLH1 proteins in endometrial carcinomas. Pol J Pathol 2010; 61: 185-191.

\section{Address for correspondence}

\section{Alp Usubutun}

Department of Pathology

Hacettepe University

06100 Ankara, Turkey

e-mail: alpusubutun@yahoo.com 